\title{
Approximation of maps into spheres by piecewise-regular maps of class $C^{k}$
}

\author{
Marcin Bilski ${ }^{1}$
}

Received: 11 December 2018 / Published online: 16 May 2019

(c) The Author(s) 2019

\begin{abstract}
The aim of this paper is to prove that every continuous map from a compact subset of a real algebraic variety into a sphere can be approximated by piecewise-regular maps of class $\mathcal{C}^{k}$, where $k$ is an arbitrary nonnegative integer.
\end{abstract}

Mathematics Subject Classification 14P05 $\cdot 14 \mathrm{P} 10 \cdot 26 \mathrm{C} 15$

\section{Introduction}

The problem of algebraic approximation of continuous maps into spheres has been studied for many years (cf. [1,2,10] and references therein).

Since regular maps are often to rigid to approximate arbitrary continuous maps (cf. $[1,3,4]$ ), it is natural to ask whether approximation by maps from larger classes is possible. One of such classes is the class of continuous rational maps (in real algebraic geometry studied systematically for the first time in [9]). On nonsingular real algebraic varieties it coincides with the class of regulous maps (also known as continuous hereditarily rational maps $\mathrm{cf}$. $[5,8]$ ). Continuous rational maps and regulous maps have attracted a lot of attention in recent years (see [5-8,10,12-14] and references therein). It has turned out, for example, that every continuous map between spheres can be approximated by continuous rational maps (see [10]). However, not every continuous map from an arbitrary compact nonsingular real algebraic variety into a sphere can be approximated by continuous rational maps (see also [10]).

Communicated by Ngaiming Mok.

Research partially supported by the NCN Grant 2014/13/B/ST1/00543.

Marcin Bilski

Marcin.Bilski@im.uj.edu.pl

1 Faculty of Mathematics and Computer Science, Jagiellonian University, Łojasiewicza 6, 30-348 Kraków, Poland 
In this paper (as in $[10,11]$ ), a real algebraic variety is, by definition, a locally ringed space isomorphic to an algebraic subset of $\mathbf{R}^{m}$, for some $m$, endowed with the Zariski topology and the sheaf of real-valued regular functions. Each real algebraic variety carries also the Euclidean topology induced by the standard metric in $\mathbf{R}$. Unless explicitly stated otherwise, all topological notions relating to real algebraic varieties refer to the Euclidean topology.

In a recent paper [11], Kucharz introduced a class of piecewise-regular maps. We say that a continuous map $f: V \supset X \rightarrow W$, where $V$ and $W$ are real algebraic varieties and $X \subset V$ is some subset, is a piecewise-regular map if the following holds. There is a stratification $\mathcal{S}$ of $V$ such that for every stratum $S \in \mathcal{S}$ the restriction of $f$ to each connected component of $S \cap X$ is a regular map. (For precise definition see Section 2.)

Under the assumption that $X$ is a compact subset of a real algebraic variety, Kucharz proved that every continuous map from $X$ into a sphere can be approximated by piecewise-regular maps (cf. [11], Theorem 1.5). Another result of [11] (see Theorem 1.6) says that if $V$ is a compact nonsingular real algebraic variety, then each continuous map from $V$ into a sphere is homotopic to a piecewise-regular map of class $\mathcal{C}^{k}$, where $k$ is an arbitrary nonnegative integer. (For $k=\infty$ this does not hold true, see [11], Ex. 1.7.)

The following question concerning generalization of Theorems 1.5, 1.6 of [11] has been asked in [11] (see the paragraph preceding Example 1.8). Let $V$ be a compact nonsingular real algebraic variety and $m, k$, be positive integers. Can every continuous map from $V$ into the $m$-dimensional unit sphere be approximated by piecewise-regular maps of class $\mathcal{C}^{k}$ ? The aim of the present paper is to answer this question in affirmative. To do this, we introduce a new concept of quasi-regulous map. It turns out that the class of quasi-regulous maps is a subclass of the class of piecewise-regular maps. We show that continuous maps from $V$ into the $m$-dimensional unit sphere can be approximated by quasi-regulous maps of class $\mathcal{C}^{k}$.

Let $W$ be a Zariski open subset of $\mathbf{R}^{n}$. Recall that a map $g: W \rightarrow \mathbf{R}^{l}$ is said to be a regulous map if it is continuous and there are polynomials $P_{1}, \ldots, P_{l}, Q_{1}, \ldots, Q_{l} \in$ $\mathbf{R}\left[T_{1}, \ldots, T_{n}\right]$ such that no $Q_{i}$ is identically equal to zero and

$$
g(x)=\left(\frac{P_{1}(x)}{Q_{1}(x)}, \ldots, \frac{P_{l}(x)}{Q_{l}(x)}\right)
$$

for all points $x \in W$ with $Q_{1}(x) \neq 0, \ldots, Q_{l}(x) \neq 0$. (As mentioned above, on nonsingular real algebraic varieties "regulous" means "continuous rational". These maps have been investigated in more general context (cf. $[5,8,10]$ ), but here it is sufficient to consider them on Zariski open subsets of $\mathbf{R}^{n}$.)

Definition Let $W$ be a Zariski open subset of $\mathbf{R}^{n}$ and let $k$ be a nonnegative integer. A function $g: W \rightarrow \mathbf{R}$ is called $\mathcal{C}^{k}$ quasi-regulous if it is of class $\mathcal{C}^{k}$ and there is a regulous function $f: W \rightarrow \mathbf{R}$ such that $|g(x)|=|f(x)|$, for every $x \in W$. Let $M$ be any (not necessarily algebraic) subset of $\mathbf{R}^{n}$. A function $\tilde{g}: M \rightarrow \mathbf{R}$ is called $\mathcal{C}^{k}$ quasi-regulous if there are a Zariski open neighborhood $W$ of $M$ in $\mathbf{R}^{n}$ and a $\mathcal{C}^{k}$ quasi-regulous function $g: W \rightarrow \mathbf{R}$ such that 
$\tilde{g}=\left.g\right|_{M}$. A map $h=\left(h_{1}, \ldots, h_{l}\right): M \rightarrow \mathbf{R}^{l}$ is called $\mathcal{C}^{k}$ quasi-regulous if $h_{i}$ is a $\mathcal{C}^{k}$ quasi-regulous function, for every $i=1, \ldots, l$.

Convention. By a quasi-regulous map we mean a $\mathcal{C}^{0}$ quasi-regulous map.

Since real algebraic varieties are, by definition, isomorphic to real algebraic sets, we may assume that $V$ in the question stated above is a real algebraic subset of some $\mathbf{R}^{n}$. Actually, we prove that every continuous map from any compact (not necessarily algebraic) subset $M$ of $\mathbf{R}^{n}$ to the unit sphere $\mathbf{S}^{m} \subset \mathbf{R}^{m+1}$ can be approximated by $\mathcal{C}^{k}$ quasi-regulous maps. As in [11], approximation is expressed in terms of the compact-open topology of the space $\mathcal{C}\left(M, \mathbf{S}^{m}\right)$ of all continuous maps from $M$ to $\mathbf{S}^{m}$. More precisely, by definition, a map $f \in \mathcal{C}\left(M, \mathbf{S}^{m}\right)$ can be approximated by $\mathcal{C}^{k}$ quasi-regulous maps if every neighborhood of $f$ (with respect to the compact-open topology) in $\mathcal{C}\left(M, \mathbf{S}^{m}\right)$ contains a $\mathcal{C}^{k}$ quasi-regulous map.

In view of Corollary 1 (see Sect. 2), the affirmative answer to the question follows immediately from Theorem 1 which is our main result.

Theorem 1 Let $M \subset \mathbf{R}^{n}$ be any compact subset and let $\mathbf{S}^{m} \subset \mathbf{R}^{m+1}$ be the $m$ dimensional unit sphere. Then for every positive integer $k$, every continuous map from $M$ to $\mathbf{S}^{m}$ can be approximated by $\mathcal{C}^{k}$ quasi-regulous maps.

The notion of $\mathcal{C}^{k}$ quasi-regulous map defined above on an arbitrary subset of any $\mathbf{R}^{n}$ has a natural extension to maps defined on arbitrary subsets of real algebraic varieties (via isomorphisms between real algebraic varieties and real algebraic subsets of $\mathbf{R}^{n}$ ). Hence, in Theorem 1, the space $\mathbf{R}^{n}$ can be replaced by a real algebraic variety.

The organization of this paper is as follows. In Sect. 2 we present some preliminary results on piecewise-regular maps and quasi-regulous maps and check the inclusion between these classes. Here we also collect basic facts on differential properties of quasi-regulous maps. In Sect. 3 the proof of Theorem 1 is given.

\section{Preliminaries}

The following generalization of the definition of regular map introduced in [11] will be useful to us.

Definition Let $V, W$ be real algebraic varieties, $X \subset V$ some (nonempty) subset, and $Z$ the Zariski closure of $X$ in $V$. A map $f: X \rightarrow W$ is said to be regular if there is a Zariski open neighborhood $Z_{0} \subseteq Z$ of $X$ and a regular map $\tilde{f}: Z_{0} \rightarrow W$ such that $\left.\tilde{f}\right|_{X}=f$.

By a stratification of a real algebraic variety $V$ we mean a finite collection of pairwise disjoint Zariski locally closed subvarieties (some possibly empty) whose union is equal to $V$.

Definition Let $V, W$ be real algebraic varieties, $f: X \rightarrow W$ a continuous map defined on some subset $X \subset V$, and $\mathcal{S}$ a stratification of $V$. The map $f$ is said to be piecewise $\mathcal{S}$-regular if for every stratum $S \in \mathcal{S}$ the restriction of $f$ to each connected component of $X \cap S$ is a regular map (when $X \cap S$ is non-empty). Moreover, $f$ is said to be piecewise-regular if it is piecewise $\mathcal{T}$-regular for some stratification $\mathcal{T}$ of $V$. 
The next theorem requires the notion of nonsingular algebraic arc (cf. [11]). Given a real algebraic variety $V$, a subset $A \subset V$ is said to be a nonsingular algebraic arc if its Zariski closure $C$ in $V$ is an algebraic curve (that is, $\operatorname{dim}(C)=1$ ), $A \subset C \backslash \operatorname{Sing}(C)$, and $A$ is homeomorhpic to $\mathbf{R}$.

The following result is taken from [11] (Theorem 2.9).

Theorem 2 Let $V, W$ be real algebraic varieties, $X \subset V$ a semialgebraic subset and $f: X \rightarrow W$ a continuous semialgebraic map. Then the following conditions are equivalent:

(a) The map $f$ is piecewise-regular

(b) For every nonsingular algebraic arc $A$ in $V$ with $A \subset X$, there exists a nonempty open subset $A_{0} \subset A$ such that $\left.f\right|_{A_{0}}$ is a regular map.

Now we prove a corollary which explains the relation between quasi-regulous maps and piecewise-regular maps.

Corollary 1 Let $M \subset \mathbf{R}^{n}$ be any subset. Every quasi-regulous map from $M$ to some $\mathbf{R}^{m}$ is a piecewise-regular map.

Proof Let $\tilde{f}: M \rightarrow \mathbf{R}^{m}$ be a quasi-regulous map. By definition, there is a quasiregulous map $f=\left(f_{1}, \ldots, f_{m}\right): X \rightarrow \mathbf{R}^{m}$ defined on a Zariski open neighborhood $X$ of $M$ in $\mathbf{R}^{n}$ such that $\left.f\right|_{M}=\tilde{f}$. We show that $f$ is piecewise-regular which clearly implies that $\tilde{f}$ is piecewise-regular too.

We check that $f$ is regular on some open subsets of nonsingular algebraic arcs and then apply Theorem 2. Let $A \subset X$ be a nonsingular algebraic arc and $C$ be the Zariski closure of $A$ in $X$. Let $g=\left(g_{1}, \ldots, g_{m}\right): X \rightarrow \mathbf{R}^{m}$ be a regulous map such that $\left|f_{i}(x)\right|=\left|g_{i}(x)\right|$ for every $i \in\{1, \ldots, m\}$ and $x \in X$.

Zariski open sets in $\mathbf{R}^{n}$ are nonsingular real algebraic varieties hence by Proposition 7 of [8] there are $P_{i}, Q_{i} \in \mathbf{R}\left[T_{1}, \ldots, T_{n}\right]$ such that $\left.g_{i}\right|_{C}=\left.\frac{P_{i}}{Q_{i}}\right|_{C}$ outside the zero-set of $Q_{i}$ and $Q_{i}$ is not identically equal to zero on $C$, for $i \stackrel{Q_{i}}{=} 1, \ldots, m$. Set $E_{i}:=$ $\left(\left.Q_{i}\right|_{C}\right)^{-1}(0) \cup\left(\left.P_{i}\right|_{C}\right)^{-1}(0)$ and observe that, for every $i$, either $A \cap E_{i}$ is finite or $A \subset E_{i}$. It is clear that in the second case, $\left.f_{i}\right|_{A}=0$. In the first one, $\left.f_{i}\right|_{A}$ is regular on every connected component of $A \backslash E_{i}$. This is because on such a component $S, f_{i}$ has constant sign (by continuity) so, either $\left.f_{i}\right|_{S}=\left.\frac{P_{i}}{Q_{i}}\right|_{S}$ or $\left.f_{i}\right|_{S}=-\left.\frac{P_{i}}{Q_{i}}\right|_{S}$, and $Q_{i}$ vanishes nowhere on $S$. Therefore the map $f$ is regular on every connected component of $A \backslash T$, where $T$ is the union of all $E_{i}$ such that $A$ is not a subset of $E_{i}$. Now the proof is complete by Theorem 2 .

The following lemmas will be used in the proof of our main result.

Lemma 1 Let $l, k \in \mathbf{N}, l, k \geq 1$.

(a) Let $f\left(y_{1}, \ldots, y_{p}\right)=\left(y_{1}^{2 k l}+\cdots+y_{p}^{2 k l}\right)^{\frac{1}{l}}$. Then $f \in \mathcal{C}^{k}\left(\mathbf{R}^{p}\right)$ and every partial derivative of $f$ of order up to $k$ vanishes at $0 \in \mathbf{R}^{p}$.

(b) Let $g(x, y)=\frac{x^{2 k} y^{2 k}}{\left(x^{2 k l}+y^{2 k l}\right)^{\frac{1}{T}}}$. Then $g \in \mathcal{C}^{k}\left(\mathbf{R}^{2}\right)$ and every partial derivative of $g$ of order up to $k$ vanishes at $0 \in \mathbf{R}^{2}$. 
(c) Let $h(x, y)=\frac{x^{4 k}}{\left(x^{2 k l}+y^{2 k l}\right)^{\frac{1}{T}}}$. Then $h \in \mathcal{C}^{k}\left(\mathbf{R}^{2}\right)$ and every partial derivative of $h$ of order up to $k$ vanishes at $0 \in \mathbf{R}^{2}$.

Proof Let us prove (a). Fix any $j \in \mathbf{N}$ with $0 \leq j \leq k$. Observe that every partial derivative of $f$ at $\left(y_{1}, \ldots, y_{p}\right) \neq(0, \ldots, 0)$ of order $j$ is the sum of terms of the following form, multiplied by constants:

$$
\left(y_{1}^{2 k l}+\cdots+y_{p}^{2 k l}\right)^{\frac{1}{l}-t} y_{1}^{(2 k l-1) s_{1}-m_{1}} \cdots y_{p}^{(2 k l-1) s_{p}-m_{p}},
$$

where $t, s_{i}, m_{i} \in \mathbf{N}$ satisfy $0 \leq t \leq j$ and $s_{1}+\cdots+s_{p}=t$, and $m_{1}+\cdots+m_{p}=j-t$. Moreover, $(2 k l-1) s_{i}-m_{i} \geq 0$, for $i=1, \ldots, p$. (By a partial derivative of $f$ of order zero we mean $f$ itself.) Clearly, every such term is an analytic function in $\left(y_{1}, \ldots, y_{p}\right)$ on $\mathbf{R}^{p} \backslash\{0\}^{p}$. We will check that every such term tends to zero as $\left(y_{1}, \ldots, y_{p}\right)$ approaches zero. This is an immediate consequence of the following inequalities.

$$
\begin{aligned}
& \left|\left(y_{1}^{2 k l}+\cdots+y_{p}^{2 k l}\right)^{\frac{1}{l}-t} y_{1}^{(2 k l-1) s_{1}-m_{1}} \cdots \cdots y_{p}^{(2 k l-1) s_{p}-m_{p}}\right| \\
& \quad \leq\left(y_{1}^{2 k l}+\cdots+y_{p}^{2 k l}\right)^{\frac{1}{l}-t}\left(\max _{i}\left|y_{i}\right|\right)^{(2 k l-1) t-j+t} \\
& \quad \leq p\left(\max _{i}\left|y_{i}\right|\right)^{2 k l \cdot\left(\frac{1}{l}-t\right)}\left(\max _{i}\left|y_{i}\right|\right)^{(2 k l t-j)}=p\left(\max _{i}\left|y_{i}\right|\right)^{(2 k-j)} .
\end{aligned}
$$

This implies that every partial derivative of $\left.f\right|_{\mathbf{R}^{p} \backslash\{0\}^{p}}$ at $\left(y_{1}, \ldots, y_{p}\right)$ of order up to $k$ tends to zero as $\left(y_{1}, \ldots, y_{p}\right)$ approaches zero.

In order to see that every partial derivative of $f$ at $(0, \ldots, 0)$ of order up to $k$ exists and equals 0 , it is sufficient to observe that when we divide the righthand side of the last inequality, for $0 \leq j \leq(k-1)$, by $\max _{i}\left|y_{i}\right|$, the obtained expression still tends to zero as $\left(y_{1}, \ldots, y_{p}\right)$ approaches zero. The proof of $(a)$ is complete.

Let us prove (b). Fix any $j \in \mathbf{N}$ with $0 \leq j \leq k$. Observe that every partial derivative of $g$ at $(x, y) \neq(0,0)$ of order $j$ is the sum of terms of the following form, multiplied by constants:

$$
\left(x^{2 k l}+y^{2 k l}\right)^{-\frac{1}{l}-t} x^{(2 k l-1) s_{1}+2 k-m_{1}} \cdot y^{(2 k l-1) s_{2}+2 k-m_{2}},
$$

where $t, s_{i}, m_{i} \in \mathbf{N}$ satisfy $0 \leq t \leq j$ and $s_{1}+s_{2}=t$, and $m_{1}+m_{2}=j-t$. Moreover, $(2 k l-1) s_{i}+2 k-m_{i} \geq 0$, for $i=1,2$. Clearly, every such term is an analytic function in $(x, y)$ on $\mathbf{R}^{2} \backslash\{0\}^{2}$. We will check that every such term tends to zero as $(x, y)$ approaches zero. This is an immediate consequence of the following inequalities.

$$
\begin{aligned}
& \left|\left(x^{2 k l}+y^{2 k l}\right)^{-\frac{1}{T}-t} x^{(2 k l-1) s_{1}+2 k-m_{1}} \cdot y^{(2 k l-1) s_{2}+2 k-m_{2}}\right| \\
& \quad \leq\left(x^{2 k l}+y^{2 k l}\right)^{-\frac{1}{l}-t} \cdot \max \{|x|,|y|\}^{(2 k l-1) t+4 k-j+t} \\
& \quad \leq \max \{|x|,|y|\}^{2 k l \cdot\left(-\frac{1}{T}-t\right)} \cdot \max \{|x|,|y|\}^{(2 k l t+4 k-j)}=\max \{|x|,|y|\}^{2 k-j} .
\end{aligned}
$$


This implies that every partial derivative of $\left.g\right|_{\mathbf{R}^{2} \backslash\{0\}^{2}}$ at $(x, y)$ of order up to $k$ tends to zero as $(x, y)$ approaches zero.

In order to see that every partial derivative of $g$ at $(0,0)$ of order up to $k$ exists and equals 0 , it is sufficient to observe that when we divide the righthand side of the last inequality, for $0 \leq j \leq(k-1)$, by $\max \{|x|,|y|\}$, the obtained expression still tends to zero as $(x, y)$ approaches zero. The proof of $(b)$ is complete.

The claim (c) can be proved in the way very similar to $(b)$.

The class of functions defined below will be used for several times in the sequel.

Definition For any open subset $U$ of $\mathbf{R}^{n}$, let $\mathcal{C}_{l}^{k}(U)$ denote the class of all functions $v: U \rightarrow \mathbf{R}$ for which $|v|^{\frac{1}{T}} \in \mathcal{C}^{k}(U)$.

Lemma 2 Let $U$ be an open subset of $\mathbf{R}^{n}$. Let $v_{1}, \ldots, v_{p} \in \mathcal{C}_{2 k l}^{k}(U)$ be nonnegative functions, where $k, l \in \mathbf{N}$ and $k, l \geq 1$. Then $v_{1}+\cdots+v_{p} \in \mathcal{C}_{l}^{k}(U)$ and $\frac{v_{1} v_{2}}{v_{1}+v_{2}} \in \mathcal{C}_{l}^{k}(U)$, and $\frac{v_{1}^{2}}{v_{1}+v_{2}} \in \mathcal{C}_{l}^{k}(U)$. Here, if $v_{1}(x)=v_{2}(x)=0$, then, by definition, $\frac{v_{1}(x) v_{2}(x)}{v_{1}(x)+v_{2}(x)}=\frac{v_{1}^{2}(x)}{v_{1}(x)+v_{2}(x)}=0$.

Proof There are $u_{1}, \ldots, u_{p} \in \mathcal{C}^{k}(U)$ such that $v_{1}=u_{1}^{2 k l}, \ldots, v_{p}=u_{p}^{2 k l}$.

The map $\left(v_{1}+\cdots+v_{p}\right)^{\frac{1}{l}}$ is the composition of the map $f\left(y_{1}, \ldots, y_{p}\right)=\left(y_{1}^{2 k l}+\right.$ $\left.\cdots+y_{p}^{2 k l}\right)^{\frac{1}{l}}$ with $x \mapsto\left(u_{1}(x), \ldots, u_{p}(x)\right)$. By Lemma $1(a)$, we have $\left(v_{1}+\cdots+\right.$ $\left.v_{p}\right)^{\frac{1}{l}} \in \mathcal{C}^{k}(U)$, hence $v_{1}+\cdots+v_{p} \in \mathcal{C}_{l}^{k}(U)$.

Similarly, the map $\left(\frac{v_{1} v_{2}}{v_{1}+v_{2}}\right)^{\frac{1}{l}}$ is the composition of ${ }^{\prime} g\left(y_{1}, y_{2}\right)=\frac{y_{1}^{2 k} y_{2}^{2 k}}{\left(y_{1}^{2 k l}+y_{2}^{2 k l}\right)^{\frac{1}{l}}}$ with $x \mapsto\left(u_{1}(x), u_{2}(x)\right)$, and the map $\left(\frac{v_{1}^{2}}{v_{1}+v_{2}}\right)^{\frac{1}{l}}$ is the composition of $h\left(y_{1}, y_{2}\right)=$ $\frac{y_{1}^{4 k}}{\left(y_{1}^{2 k l}+y_{2}^{2 k l}\right)^{\frac{1}{T}}}$ with $x \mapsto\left(u_{1}(x), u_{2}(x)\right)$. Therefore, by Lemma $1(b),(c)$, the proof is complete.

Lemma 3 Let $U$ be an open subset of $\mathbf{R}^{n}$. Let $f \in \mathcal{C}_{l}^{k}(U)$, where $l, k \in \mathbf{N}$ with $k \geq 1$ and $l \geq k+1$. Then for every continuous function $g: U \rightarrow \mathbf{R}$ such that for every $x \in U$ either $g(x)=f(x)$ or $g(x)=-f(x)$, we have $g \in \mathcal{C}^{k}(U)$.

Proof By hypothesis, there is $h \in \mathcal{C}^{k}(U)$ such that $h^{l}=|f|=|g|$. In particular, $h^{-1}(0)=f^{-1}(0)=g^{-1}(0)$. By continuity of $g$, for every connected component $A_{i}$ of $U \backslash\left(h^{-1}(0)\right)$ there is $\beta_{i} \in\{-1,1\}$ with $g=\beta_{i} \cdot h^{l}$ on $A_{i}$ and $\left.g\right|_{h^{-1}(0)}=0$.

Now, it is not difficult to observe that for such $g$ there exist all partial derivatives of order up to $k$ at every point of $U$. These partial derivatives vanish identically on $h^{-1}(0)$ and are continuous on $U$.

Let $N$ be any compact subset of $\mathbf{R}^{n}$ and let $h: N \rightarrow \mathbf{R}$ be a continuous function. For any $\delta>0$ put $N_{\delta}=\left\{x \in \mathbf{R}^{n}: \operatorname{dist}(x, N)<\delta\right\}$ and put $\|h\|_{N}=\sup _{x \in N}|h(x)|$. We assume $\emptyset_{\delta}=\varnothing$ and $\|h\|_{\varnothing}=0$.

Lemma 4 Let $G \subset \subset \mathbf{R}^{n}$ be an open ball and let $f, g: \bar{G} \rightarrow \mathbf{R}$ be continuous, nonnegative, semialgebraic functions. Assume that $(f \cdot g)^{-1}(0)$ is a compact $\mathcal{C}^{\infty}$ 
submanifold of $G$ of pure codimension 1. Then for every $\varepsilon>0$ there are $P, Q \in$ $\mathbf{R}\left[x_{1}, \ldots, x_{n}\right]$ and an open tubular neighborhood $T \subset \subset G$ of $(f \cdot g)^{-1}(0)$ of constant radius such that $\|f \cdot g\|_{T}<\varepsilon,(P \cdot Q)^{-1}(0) \cap \partial G=\emptyset$ and such that the following conditions are satisfied:

(a) for every connected component $A$ of $G \backslash(f \cdot g)^{-1}(0)$ there is a connected component $B$ of $G \backslash(P \cdot Q)^{-1}(0)$ with $A \subset B \cup \bar{T}$. Moreover, for every connected component $B$ of $G \backslash(P \cdot Q)^{-1}(0)$, there is at most one connected component $A$ of $G \backslash(f \cdot g)^{-1}(0)$ with $A \subset B \cup \bar{T}$.

(b) for every $l \in \mathbf{N}$ there are $\tilde{f}, \tilde{g} \in \mathbf{R}\left[x_{1}, \ldots, x_{n}\right], \tilde{f}, \tilde{g}>0$ on $\mathbf{R}^{n}$, such that $\left\|f-\tilde{f} \cdot P^{2 l}\right\|_{G} \leq \varepsilon,\left\|g-\tilde{g} \cdot Q^{2 l}\right\|_{G} \leq \varepsilon$.

Remark The algebraicity assumptions above can be relaxed, but we prefer to state this lemma in the setting in which it will be used.

Proof of Lemma 4 Fix $\varepsilon>0$ (we may assume $\varepsilon<1$ ). Choose an open tubular neighborhood $T$ of $(f \cdot g)^{-1}(0)$ relatively compact in $G$ of constant radius so small that $\|f \cdot g\|_{T}<\varepsilon$ and for every connected component $A$ of $G \backslash(f \cdot g)^{-1}(0)$ there is precisely one connected component $\hat{A}$ of $G \backslash \bar{T}$ such that $\hat{A} \subset A$.

Next choose $\delta>0$ so small that $\left(f^{-1}(0)\right)_{2 \delta} \cup\left(g^{-1}(0)\right)_{2 \delta} \subset T$, and moreover, $\|f\|_{\left(f^{-1}(0)\right)_{2 \delta}}<\frac{\varepsilon}{2}$ and $\|g\|_{\left(g^{-1}(0)\right)_{2 \delta}}<\frac{\varepsilon}{2}$.

Now there is $0<\varepsilon_{1}<1$ such that $\left\{x \in \bar{G}: f(x) \leq \varepsilon_{1}\right\} \subset\left(f^{-1}(0)\right)_{\delta}$ and $\left\{x \in \bar{G}: g(x) \leq \varepsilon_{1}\right\} \subset\left(g^{-1}(0)\right)_{\delta}$. Using the Stone-Weierstrass theorem, approximate $f-\frac{\varepsilon_{1}}{2}$ and $g-\frac{\varepsilon_{1}}{2}$ on $\bar{G}$ by polynomials $P, Q$, respectively, with precision high enough to ensure that $P^{-1}(0) \cap \bar{G} \subset\left(f^{-1}(0)\right)_{\delta}, Q^{-1}(0) \cap \bar{G} \subset\left(g^{-1}(0)\right)_{\delta},\left.P\right|_{f^{-1}(0)}<0$, $\left.Q\right|_{g^{-1}(0)}<0,\left.P\right|_{\bar{G} \backslash\left(f^{-1}(0)\right)_{\delta}}>0,\left.Q\right|_{\bar{G} \backslash\left(g^{-1}(0)\right)_{\delta}}>0$. In particular, it follows that $(P \cdot Q)^{-1}(0) \cap G \subset T$ and $(P \cdot Q)^{-1}(0) \cap \partial G=\emptyset$.

Proof of (a) Let $\hat{A}_{1}, \ldots, \hat{A}_{s}$ denote all pairwise distinct connected components of $G \backslash \bar{T}$. By the previous paragraph, $\hat{A}_{j} \cap(P \cdot Q)^{-1}(0)=\emptyset$, so there is the uniquely determined connected component $B_{j}$ of $G \backslash(P \cdot Q)^{-1}(0)$ with $\hat{A}_{j} \subset B_{j}$, for $j=$ $1, \ldots, s$.

We shall need the following property of $\left\{B_{i}\right\}$. If $i \neq j$, then $B_{i} \neq B_{j}$. If this was not true, then there were $a_{i} \in \hat{A}_{i}$ and $a_{j} \in \hat{A}_{j}$ and a path connecting these points in $B_{i}=B_{j}$. The path must intersect $(f \cdot g)^{-1}(0)$ at some point $b$ because $\hat{A}_{i}, \hat{A}_{j}$ are contained in two distinct connected components of $G \backslash(f \cdot g)^{-1}(0)$. Then either $f(b)=0$ or $g(b)=0$. Assume that $f(b)=0$. (For $g(b)=0$ we proceed identically.) We have $P(b)<0$ and $P\left(a_{i}\right)>0$. Consequently, at some point $d$ of the path $P(d)=0$. This implies that $B_{i} \cap(P \cdot Q)^{-1}(0) \neq \emptyset$, a contradiction.

Now we show that for every connected component $B$ of $G \backslash(P \cdot Q)^{-1}(0)$, there is at most one connected component $A$ of $G \backslash(f \cdot g)^{-1}(0)$ such that $A \subset B \cup \bar{T}$. Fix $B$. Observe that for every $i \in\{1, \ldots, s\}$ either $\hat{A}_{i} \subset B$ or $\hat{A}_{i} \cap B=\emptyset$. Indeed, if for some $i$ we had $\hat{A}_{i} \cap B \neq \emptyset$ and $\hat{A}_{i}$ was not a subset of $B$, then from the connectedness of $\hat{A}_{i}$ we had $\partial B \cap \hat{A}_{i} \neq \emptyset$. But this is impossible because $\left.(P \cdot Q)\right|_{\partial B \cap G}=0$ and $(P \cdot Q)^{-1}(0) \cap \hat{A}_{i}=\emptyset$.

Suppose that $\hat{A}_{i} \cup \hat{A}_{j} \subset B \cup \bar{T}$ for $i \neq j$. We know that $\hat{A}_{i} \cup \hat{A}_{j}$ is not a subset of $B$ so, in view of previous paragraph, either $\hat{A}_{i} \subset \bar{T}$ or $\hat{A}_{j} \subset \bar{T}$, a contradiction. 
Let us verify the first claim of ( $a$ ). Fix a connected component $A$ of $G \backslash(f \cdot g)^{-1}(0)$. Let $\hat{A}$ be the unique connected component of $G \backslash \bar{T}$ such that $\hat{A} \subset A$. Since $(P$. $Q)^{-1}(0) \cap \hat{A}=\varnothing$, there is the uniquely determined connected component $B$ of $G \backslash(P \cdot Q)^{-1}(0)$ containing $\hat{A}$. Therefore $\hat{A} \cup \bar{T} \subset B \cup \bar{T}$ and the proof of $(a)$ is complete because $A \subset \hat{A} \cup \bar{T}$.

Proof of (b) Fix any $l \in \mathbf{N}$. Since $\|f\|_{\left(f^{-1}(0)\right)_{\delta}}<\frac{\varepsilon}{2}$ and $\|g\|_{\left(g^{-1}(0)\right)_{\delta}}<\frac{\varepsilon}{2}$, we can take $\alpha \in \mathbf{R}, \alpha>0$ so small that

$$
\left\|\frac{f+\alpha}{P^{2 l}+\alpha} \cdot P^{2 l}\right\|_{\left(f^{-1}(0)\right)_{\delta}}<\frac{\varepsilon}{2} \text { and }\left\|\frac{g+\alpha}{Q^{2 l}+\alpha} \cdot Q^{2 l}\right\|_{\left(g^{-1}(0)\right)_{\delta}}<\frac{\varepsilon}{2} .
$$

Since $P^{-1}(0) \cap G \subset\left(f^{-1}(0)\right)_{\delta}$ and $Q^{-1}(0) \cap G \subset\left(g^{-1}(0)\right)_{\delta}$ we can additionally require that

$$
\left\|\frac{f+\alpha}{P^{2 l}+\alpha} \cdot P^{2 l}-f\right\|_{G \backslash\left(f^{-1}(0)\right)_{\delta}}<\frac{\varepsilon}{2} \text { and }\left\|\frac{g+\alpha}{Q^{2 l}+\alpha} \cdot Q^{2 l}-g\right\|_{G \backslash\left(g^{-1}(0)\right)_{\delta}}<\frac{\varepsilon}{2} .
$$

Note that $f+\alpha, g+\alpha>0$, hence for close polynomial approximations $\bar{f}, \bar{g}$ of $\sqrt{\frac{f+\alpha}{P^{2 l}+\alpha}}, \sqrt{\frac{g+\alpha}{Q^{2 l}+\alpha}}$ on $\bar{G}$, after defining $\tilde{f}:=\bar{f}^{2}+\eta, \tilde{g}:=\bar{g}^{2}+\eta$, for small $\eta \in \mathbf{R}, \eta>0$, we have polynomial approximations $\tilde{f}, \tilde{g}$ of $\frac{f+\alpha}{P^{2 l}+\alpha}, \frac{g+\alpha}{Q^{2 l}+\alpha}$ on $\bar{G}$ with $\tilde{f}>0, \tilde{g}>0$ on $\mathbf{R}^{n}$ and

$$
\left\|\tilde{f} \cdot P^{2 l}\right\|_{\left(f^{-1}(0)\right)_{\delta}}<\frac{\varepsilon}{2},\left\|\tilde{g} \cdot Q^{2 l}\right\|_{\left(g^{-1}(0)\right)_{\delta}}<\frac{\varepsilon}{2}
$$

and

$$
\left\|\tilde{f} \cdot P^{2 l}-f\right\|_{G \backslash\left(f^{-1}(0)\right)_{\delta}}<\frac{\varepsilon}{2},\left\|\tilde{g} \cdot Q^{2 l}-g\right\|_{G \backslash\left(g^{-1}(0)\right)_{\delta}}<\frac{\varepsilon}{2} .
$$

Finally observe that

$$
\begin{aligned}
& \left\|f-\tilde{f} \cdot P^{2 l}\right\|_{G} \leq \max \left\{\left\|f-\tilde{f} \cdot P^{2 l}\right\|_{\left(f^{-1}(0)\right)_{\delta}},\left\|f-\tilde{f} \cdot P^{2 l}\right\|_{G \backslash\left(f^{-1}(0)\right)_{\delta}}\right\} \\
& \quad \leq \max \left\{\|f\|_{\left(f^{-1}(0)\right)_{\delta}}+\left\|\tilde{f} \cdot P^{2 l}\right\|_{\left(f^{-1}(0)\right)_{\delta}}, \frac{\varepsilon}{2}\right\} \leq \varepsilon .
\end{aligned}
$$

The same argument shows that $\left\|g-\tilde{g} \cdot Q^{2 l}\right\|_{G} \leq \varepsilon$, proving (b).

\section{Proof of Theorem 1}

Let $u=\left(u_{1}, \ldots, u_{m+1}\right): M \rightarrow \mathbf{S}^{m} \subset \mathbf{R}^{m+1}$ be a continuous map ( $\left.m \geq 1\right)$. In particular, $u_{1}^{2}+\cdots+u_{m+1}^{2}=1$. We will show that for every positive integer $k, u$ can be approximated by $\mathcal{C}^{k}$ quasi-regulous maps from $M$ to $\mathbf{S}^{m} \subset \mathbf{R}^{m+1}$. 
First we show that there is a Nash (i.e. both semi-algebraic and analytic) map $\tilde{u}=\left(\tilde{u}_{1}, \ldots, \tilde{u}_{m+1}\right)$ from $\mathbf{R}^{n} \backslash \Sigma \supset M$ to $\mathbf{S}^{m}$, where $\Sigma$ is a compact algebraic subset of $\mathbf{R}^{n}$ of codimension at least 2 , with the following properties. The map $\tilde{u}$ approximates $u$ on $M$ and, for every $i=1, \ldots, m+1, \tilde{u}_{i}^{-1}(0) \cup \Sigma$ is a compact $\mathcal{C}^{\infty}$ submanifold of $\mathbf{R}^{n}$ of pure dimension $n-1$ or $\tilde{u}_{i}^{-1}(0) \cap M=\emptyset$.

To do this, apply the Stone-Weierstrass theorem to approximate $u_{1}, \ldots, u_{m+1}$ by polynomials $\hat{u}_{1}, \ldots, \hat{u}_{m+1}$ on $M$. Next choose $\varepsilon \in(0,+\infty)$ small enough and a positive integer $\hat{m}$ large enough to ensure that after having replaced every $\hat{u}_{i}$ by $\hat{u}_{i}+\varepsilon \cdot\left(x_{1}^{2}+\cdots+x_{n}^{2}\right)^{\hat{m}}$ we obtain a map to $\mathbf{R}^{m+1}$ with the following property. There is an open ball $G \subset \subset \mathbf{R}^{n}$ such that for every $i, \hat{u}_{i}$ is bounded from below by 1 outside $G$ and $\hat{u}_{i}$ still approximates $u_{i}$ on $M$.

Next, add small constants to all $\hat{u}_{i}$ 's to ensure that the zero-set of every $\hat{u}_{i}$ is a compact $\mathcal{C}^{\infty}$ submanifold of $G$ of pure dimension $n-1$ or is empty and that $\operatorname{dim}\left(\hat{u}_{1}^{-1}(0) \cap \ldots \cap \hat{u}_{m+1}^{-1}(0)\right) \leq n-2$. Define $\Sigma:=\hat{u}_{1}^{-1}(0) \cap \cdots \cap \hat{u}_{m+1}^{-1}(0)$ and observe that if the approximation is close enough, then $\Sigma \cap M=\emptyset$.

Finally, set $\hat{u}=\left(\hat{u}_{1}, \ldots, \hat{u}_{m+1}\right)$ and compose $\left.\hat{u}\right|_{\mathbf{R}^{n} \backslash \Sigma}$ with the standard retraction of $\mathbf{R}^{m+1} \backslash\{0\}^{m+1}$ onto $\mathbf{S}^{m}$, to obtain

$$
\tilde{u}=\left(\frac{\hat{u}_{1}}{\sqrt{\hat{u}_{1}^{2}+\cdots+\hat{u}_{m+1}^{2}}}, \ldots, \frac{\hat{u}_{m+1}}{\sqrt{\hat{u}_{1}^{2}+\cdots+\hat{u}_{m+1}^{2}}}\right) .
$$

To complete the proof of Theorem 1 it is sufficient to approximate $\tilde{u}$ on $M$ by $\mathcal{C}^{k}$ quasi-regulous maps into $\mathbf{S}^{m}$. Hence, we may assume that $u$ is a Nash map from $\mathbf{R}^{n} \backslash \Sigma \supset M$ to $\mathbf{S}^{m}$, where $\Sigma$ is a compact algebraic subset of $\mathbf{R}^{n}$ with $\operatorname{dim}(\Sigma) \leq n-2$. Moreover, for every $i=1, \ldots, m+1, u_{i}^{-1}(0) \cup \Sigma$ is a compact $\mathcal{C}^{\infty}$ submanifold of $\mathbf{R}^{n}$ of pure dimension $n-1$ or $u_{i}^{-1}(0) \cap M=\emptyset$.

If, for some $i, u_{i}^{-1}(0) \cap M=\emptyset$, then $\left.u\right|_{M}$ is a non-surjective map to $\mathbf{S}^{m}$ which can be easily approximated by regular maps to $\mathbf{S}^{m}$ because the unit sphere with a point removed is biregularly isomorphic to $\mathbf{R}^{m}$. If, for every $i, u_{i}^{-1}(0) \cup \Sigma$ is a $\operatorname{compact} \mathcal{C}^{\infty}$ submanifold of $\mathbf{R}^{n}$ of pure dimension $n-1$, then we proceed as follows.

Let us define a new family of continuous, semialgebraic non-negative functions from $\mathbf{R}^{n} \backslash \Sigma$ to $\mathbf{R}$ :

$v_{1}=\left|u_{1}\right|$,

$v_{i+1}=\sqrt{v_{i}^{2}+u_{i+1}^{2}}, i=1, \ldots, m$.

In particular, $v_{m+1}=1$. It is clear that $u_{i}, v_{i}$ satisfy the following system of equations:

$u_{1}^{2}+u_{2}^{2}=v_{2}^{2}$,

$v_{i}^{2}+u_{i+1}^{2}=v_{i+1}^{2}$, for $i=2, \ldots, m$.

We will approximate $u_{1}$ on $M$ by polynomials and, for $i \geq 2, u_{i}, v_{i}$ on $M$ by $\mathcal{C}^{k}$ quasi-regulous functions $\bar{u}_{i}, \bar{v}_{i}$ from $\mathbf{R}^{n}$ to $\mathbf{R}$ satisfying 
$\bar{u}_{1}^{2}+\bar{u}_{2}^{2}=\bar{v}_{2}^{2}$,

$\bar{v}_{i}^{2}+\bar{u}_{i+1}^{2}=\bar{v}_{i+1}^{2}$, for $i=2, \ldots, m$,

and such that $\bar{v}_{m+1}^{-1}(0)$ is an algebraic subset of $\mathbf{R}^{n}$. Since $v_{m+1}=1$, we will have $M \cap \bar{v}_{m+1}^{-1}(0)=\emptyset$ for $\bar{v}_{m+1}$ close to $v_{m+1}$ on $M$. Then, the map $w$ whose components $w_{i}$, for $i=1, \ldots, m+1$, are defined by $w_{i}=\frac{\bar{u}_{i}}{\bar{v}_{m+1}}$, is a $\mathcal{C}^{k}$ quasi-regulous map from $\mathbf{R}^{n} \backslash \bar{v}_{m+1}^{-1}(0)$ to $\mathbf{S}^{m}$ approximating $u$ on $M$, and the proof will be completed.

Now we will prove that $u_{1}$ can be approximated by polynomials and, for $i \geq 2, v_{i}$ can be approximated by regulous functions of class $\mathcal{C}^{k}$ and $u_{i}$ can be approximated by $\mathcal{C}^{k}$ quasi-regulous functions satisfying the equations listed above. This will be done indirectly: approximating every $u_{i}$, for $i=2, \ldots, m+1$, will be preceded by approximating the absolute value $\left|u_{i}\right|$ on $M$ by a nonnegative regulous function $\left|\bar{u}_{i}\right| \in \mathcal{C}_{l}^{k+1}\left(\mathbf{R}^{n}\right)$, for some $l \geq k+1$. (For the definition of $\mathcal{C}_{l}^{k+1}\left(\mathbf{R}^{n}\right)$ see Sect. 2.) Using $\left|\bar{u}_{i}\right|$ and Lemma 3, we will produce a quasi-regulous approximation of $u_{i}$ satisfying the requirements.

The whole construction will be carried out in $m$ steps. In the first step we handle the functions appearing in the equation $u_{1}^{2}+u_{2}^{2}=v_{2}^{2}$. In step number $i$, for $i \geq 2$, we deal with the functions appearing in $v_{i}^{2}+u_{i+1}^{2}=v_{i+1}^{2}$ making use of the data obtained previously.

Let us discuss the first step. From the first equation we have $\left|u_{2}\right|=\sqrt{v_{2}-u_{1}}$. $\sqrt{v_{2}+u_{1}}$ on $\mathbf{R}^{n} \backslash \Sigma$. Let $f, g: \mathbf{R}^{n} \rightarrow \mathbf{R}$ be continuous nonnegative semialgebraic functions with the following properties:

$$
\begin{aligned}
& \left.f\right|_{M}=\left.\left(\sqrt{v_{2}-u_{1}}\right)\right|_{M}, f^{-1}(0)=\left(\sqrt{v_{2}-u_{1}}\right)^{-1}(0) \cup \Sigma, \\
& \left.g\right|_{M}=\left.\left(\sqrt{v_{2}+u_{1}}\right)\right|_{M}, g^{-1}(0)=\left(\sqrt{v_{2}+u_{1}}\right)^{-1}(0) \cup \Sigma .
\end{aligned}
$$

Such functions can be obtained as follows. By the semialgebraic version of the Urysohn lemma there are a neighborhood $U$ of $\Sigma$ in $\mathbf{R}^{n}$ disjoint from $M$ and a continuous semialgebraic function $\beta: \mathbf{R}^{n} \rightarrow[0,1]$ with $\beta^{-1}(0)=\Sigma$ and $\left.\beta\right|_{\mathbf{R}^{n} \backslash U}=1$. Then, by Proposition 2.6.4 of [1], there exists an integer $N$ such that $f=\sqrt{v_{2}-u_{1}} \cdot \beta^{N}$, $g=\sqrt{v_{2}+u_{1}} \cdot \beta^{N}$ extended by zero on $\Sigma$ are continuous on $\mathbf{R}^{n}$.

Since $(f \cdot g)^{-1}(0)=u_{2}^{-1}(0) \cup \Sigma$ is a compact $\mathcal{C}^{\infty}$ submanifold of $\mathbf{R}^{n}$ of pure dimension $n-1$, there is an open ball $G \subset \subset \mathbf{R}^{n}$ such that $(f \cdot g)^{-1}(0) \cup M \subset G$. Then, clearly, $\left.f\right|_{\bar{G}},\left.g\right|_{\bar{G}}$ satisfy the hypotheses of Lemma 4.

By Lemma 4, we obtain polynomial approximations $\hat{f}$ of $f$ and $\hat{g}$ of $g$ on $\bar{G}$ of the form $\hat{f}=\tilde{f} \cdot P^{2 l}$ and $\hat{g}=\tilde{g} \cdot Q^{2 l}$ with any given precision $\varepsilon$, where $P, Q, \tilde{f}, \tilde{g} \in$ $\mathbf{R}\left[x_{1}, \ldots, x_{n}\right], \tilde{f}>0, \tilde{g}>0$ on $\mathbf{R}^{n}$ and $l=4^{m-1}(k+1)^{2 m+1}$. Define $\bar{u}_{1}, \bar{v}_{2}$ by the equations $\hat{f}^{2}=\bar{v}_{2}-\bar{u}_{1}, \hat{g}^{2}=\bar{v}_{2}+\bar{u}_{1}$ to obtain $\bar{v}_{2}=\frac{\hat{f}^{2}+\hat{g}^{2}}{2}, \bar{u}_{1}=\frac{-\hat{f}^{2}+\hat{g}^{2}}{2}$ and set $\left|\bar{u}_{2}\right|=\hat{f} \cdot \hat{g}$. Then $\bar{u}_{1}^{2}+\left|\bar{u}_{2}\right|^{2}=\bar{v}_{2}^{2}$. Note that $\bar{u}_{1}^{-1}(0)$ and $\left|\bar{u}_{2}\right|^{-1}(0)$ are algebraic subsets of $\mathbf{R}^{n}$. It is clear that $\bar{u}_{1},\left|\bar{u}_{2}\right|, \bar{v}_{2}$ approximate $u_{1},\left|u_{2}\right|, v_{2}$, respectively on $M$. To complete the first step it remains to recover $\bar{u}_{2}$ with certain regularity properties when $\left|\bar{u}_{2}\right|$ is given. Actually, we need to control some regularity properties of $\bar{v}_{2}$ as well because $\bar{v}_{2}$ will be used to define $\bar{u}_{3}$ in the next step of the construction. 
We have $\hat{f}^{2}, \hat{g}^{2} \in \mathcal{C}_{2 \cdot 4^{m-1}(k+1)^{2 m+1}}^{k+1}\left(\mathbf{R}^{n}\right)$ which, in view of Lemma 2, implies $\bar{v}_{2} \in$ $\mathcal{C}_{4^{m-1}(k+1)^{2 m}}^{k+1}\left(\mathbf{R}^{n}\right)$ and $\left|\bar{u}_{2}\right| \in \mathcal{C}_{4^{m-1}(k+1)^{2 m+1}}^{k+1}\left(\mathbf{R}^{n}\right) \subset \mathcal{C}_{4^{m-1}(k+1)^{2 m}}^{k+1}\left(\mathbf{R}^{n}\right)$.

In order to define $\bar{u}_{2}$, given $\left|\bar{u}_{2}\right|$, it is sufficient to define the sign of $\bar{u}_{2}$ on every connected component of $\mathbf{R}^{n} \backslash(P \cdot Q)^{-1}(0)$. Let $A_{1}, \ldots, A_{s}$ denote all pairwise distinct connected components of $G \backslash(f \cdot g)^{-1}(0)$. By Lemma 4, there is a tubular neighborhood $T \subset \subset G$ of $(f \cdot g)^{-1}(0)$ and there are connected components $B_{1}, \ldots, B_{s}$ of $G \backslash(P$. $Q)^{-1}(0)$ with $A_{i} \subset B_{i} \cup \bar{T}$ and such that $B_{i} \neq B_{j}$ if $i \neq j$.

Recall that $u_{2}^{-1}(0) \cup \Sigma=(f \cdot g)^{-1}(0)$ and $\operatorname{set} \operatorname{sgn}\left(\left.\bar{u}_{2}\right|_{B_{i}}\right)=\operatorname{sgn}\left(\left.u_{2}\right|_{A_{i}}\right)$, for $i=1, \ldots, s$. On every component of $G \backslash(P \cdot Q)^{-1}(0)$ different from $B_{1}, \ldots, B_{s}$ set the sign of $\bar{u}_{2}$ arbitrarily and set $\left.\bar{u}_{2}\right|_{(P \cdot Q)^{-1}(0)}=0$. Since $(P \cdot Q)^{-1}(0) \cap \partial G=\emptyset$, there is precisely one connected component $C$ of $G \backslash(P \cdot Q)^{-1}(0)$ with $\bar{C} \cap \partial G \neq \emptyset$. Therefore on every connected component $E$ of $\left(\mathbf{R}^{n} \backslash G\right) \backslash(P \cdot Q)^{-1}(0)$ we can define $\operatorname{sgn}\left(\left.\bar{u}_{2}\right|_{E}\right)=\operatorname{sgn}\left(\left.\bar{u}_{2}\right|_{C}\right)$. Clearly, $\bar{u}_{2}$ is a quasi-regulous function and, by Lemma 3 , $\bar{u}_{2} \in \mathcal{C}^{k+1}\left(\mathbf{R}^{n}\right)$.

Let us check that $\bar{u}_{2}$ approximates $u_{2}$ on $B_{i} \cap M$, for $i=1, \ldots, s$. It is clear that $\bar{u}_{2}$ approximates $u_{2}$ on $A_{i} \cap B_{i} \cap M$. This is because $u_{2}$ and $\bar{u}_{2}$ have the same sign at every point of $A_{i} \cap B_{i}$ and $\left|\bar{u}_{2}\right|$ approximates $\left|u_{2}\right|$ on $M$. On the other hand, $B_{i} \backslash A_{i} \subset \bar{T}$. If this was not true, then we had $\left(\left(B_{i} \backslash A_{i}\right) \backslash \bar{T}\right) \cap A_{j} \neq \emptyset$ for some $j \neq i$ because $\bigcup_{i=1}^{s} A_{i} \cup \bar{T}=G$. Then $\left(\left(B_{i} \backslash A_{i}\right) \backslash \bar{T}\right) \cap\left(B_{j} \cup \bar{T}\right) \neq \varnothing$ so $B_{i} \cap B_{j} \neq \varnothing$ for some $j \neq i$, a contradiction. Since $B_{i} \backslash A_{i} \subset \bar{T}$, the function $\bar{u}_{2}$ approximates $u_{2}$ on $\left(B_{i} \backslash A_{i}\right) \cap M$ because $\left|u_{2}\right|_{M \cap \bar{T}}|=(f \cdot g)|_{M \cap \bar{T}}$, and $\left|\bar{u}_{2}\right|_{M \cap \bar{T}} \mid$ approximates $\left|u_{2}\right|_{M \cap \bar{T}} \mid$, and, by Lemma $4,\left.(f \cdot g)\right|_{M \cap \bar{T}}$ may be assumed as close to zero as we wish. Hence, $\left.u_{2}\right|_{M \cap \bar{T}}$ and $\left.\bar{u}_{2}\right|_{M \cap \bar{T}}$ are close to zero therefore close to each other (even if $u_{2}$ and $\bar{u}_{2}$ have different signs at some points of $M \cap \bar{T})$.

Let us check that $\bar{u}_{2}$ approximates $u_{2}$ on $M \backslash \bigcup_{i=1}^{s} B_{i}$. Observe that $G \backslash \bigcup_{i=1}^{S} B_{i} \subset$ $\bar{T}$. This is because $\bigcup_{i=1}^{s} A_{i} \cup \bar{T}=G$ and, for every $i$, we have $A_{i} \subset B_{i} \cup \bar{T}$. Consequently, $M \backslash \bigcup_{i=1}^{S} B_{i} \subset \bar{T}$ and the assertion follows as above. Thus we have proved that $\bar{u}_{2}$ approximates $u_{2}$ on $M$.

Steps numbered by $i \in\{2, \ldots, m\}$ are slightly different. We will describe them inductively. Assume that step number $j$, where $1 \leq j<m$, has been accomplished i.e. we have continuous functions $\bar{u}_{j+1}, \bar{v}_{j+1}$ approximating $u_{j+1}$, $v_{j+1}$, respectively, on $M$ such that $\left|\bar{u}_{j+1}\right|, \bar{v}_{j+1} \in \mathcal{C}_{4^{m-j}(k+1)^{2(m-j+1)}}^{k+1}\left(\mathbf{R}^{n}\right)$ are nonnegative regulous functions satisfying $\bar{v}_{j}^{2}+\bar{u}_{j+1}^{2}=\bar{v}_{j+1}^{2}$ (we assume $\bar{v}_{1}=\left|\bar{u}_{1}\right|$ ). In particular, $\bar{u}_{j+1}$ is a quasi-regulous function and, by Lemma $3, \bar{u}_{j+1}, \bar{v}_{j+1} \in$ $\mathcal{C}^{k+1}\left(\mathbf{R}^{n}\right)$.

We will define continuous functions $\bar{u}_{j+2}, \bar{v}_{j+2}$ approximating $u_{j+2}, v_{j+2}$, respectively, on $M$ such that $\left|\bar{u}_{j+2}\right|, \bar{v}_{j+2} \in \mathcal{C}_{4^{m-j-1}(k+1)^{2(m-j)}}^{k+1}\left(\mathbf{R}^{n}\right)$ are non-negative regulous functions satisfying $\bar{v}_{j+1}^{2}+\bar{u}_{j+2}^{2}=\bar{v}_{j+2}^{2}$. In particular, $\bar{u}_{j+2}$ will be a quasi-regulous function and, by Lemma $3, \bar{u}_{j+2}, \bar{v}_{j+2}$ will be of class $\mathcal{C}^{k+1}$, which will complete the proof. 
From the input data we have semialgebraic functions $v_{j+1}, u_{j+2}, v_{j+2}$, from $\mathbf{R}^{n} \backslash \Sigma$ to $\mathbf{R}$, satisfying $v_{j+1}^{2}+u_{j+2}^{2}=v_{j+2}^{2}$. Let us denote

$$
2 \alpha=\sqrt{v_{j+2}-v_{j+1}}\left(\sqrt{v_{j+2}-v_{j+1}}+\sqrt{v_{j+2}+v_{j+1}}\right)+v_{j+1} .
$$

This is well defined on $\mathbf{R}^{n} \backslash \Sigma$ as, by the definition of $v_{j+2}$, we have $v_{j+2} \geq v_{j+1} \geq 0$. Observe that $2 \alpha=v_{j+2}+\left|u_{j+2}\right|$ so $\left|u_{j+2}\right|=\alpha-\frac{v_{j+1}^{2}}{4 \alpha}$ and $v_{j+2}=\alpha+\frac{v_{j+1}^{2}}{4 \alpha}$.

From the equation $v_{j+1}^{2}+u_{j+2}^{2}=v_{j+2}^{2}$ and from the fact that $v_{j+2} \geq v_{j+1} \geq 0$ it follows that $\left(\sqrt{v_{j+2}-v_{j+1}}\right)^{-1}(0)=u_{j+2}^{-1}(0)$. Let $f: \mathbf{R}^{n} \rightarrow \mathbf{R}$ be a continuous nonnegative semialgebraic function with the following properties: $\left.f\right|_{M}=$ $\left.\left(\sqrt{v_{j+2}-v_{j+1}}\right)\right|_{M}, f^{-1}(0)=\left(\sqrt{v_{j+2}-v_{j+1}}\right)^{-1}(0) \cup \Sigma$. Such a function can be obtained in the same way as in the first step.

Since $f^{-1}(0)=u_{j+2}^{-1}(0) \cup \Sigma$ is a $\operatorname{compact} \mathcal{C}^{\infty}$ submanifold of $\mathbf{R}^{n}$ of pure dimension $n-1$, there is an open ball $G \subset \subset \mathbf{R}^{n}$ such that $f^{-1}(0) \cup M \subset G$. Then, $\left.f\right|_{\bar{G}}$ and $\left.g\right|_{\bar{G}}=1$ satisfy the hypotheses of Lemma 4 .

By Lemma 4, we obtain polynomial approximation $\bar{\gamma}$ of $f$ on $\bar{G}$ of the form $\bar{\gamma}=\tilde{f} \cdot P^{2 l}$ with any given precision $\varepsilon$ (we assume $\varepsilon<1$ ), where $P, \tilde{f} \in$ $\mathbf{R}\left[x_{1}, \ldots, x_{n}\right], \tilde{f}>0$ on $\mathbf{R}^{n}$ and $l=4^{m-j}(k+1)^{2(m-j+1)}$. In particular, we have $\bar{\gamma} \in \mathcal{C}_{4^{m-j}(k+1)^{2(m-j+1)}}^{k+1}\left(\mathbf{R}^{n}\right)$. Next approximate, using the Stone-Weierstrass approximation theorem, the function $\left(\sqrt{v_{j+2}-v_{j+1}}+\sqrt{v_{j+2}+v_{j+1}}\right)$ on $M$ by a polynomial $\bar{A}$ positive on $\mathbf{R}^{n}$ and define $2 \bar{\alpha}:=\bar{\gamma} \bar{A}+\bar{v}_{j+1}$ on $\mathbf{R}^{n}$. Finally define $\left|\bar{u}_{j+2}\right|:=\bar{\alpha}-\frac{\bar{v}_{j+1}^{2}}{4 \bar{\alpha}}$ and $\bar{v}_{j+2}:=\bar{\alpha}+\frac{\bar{v}_{j+1}^{2}}{4 \bar{\alpha}}$.

Using Lemma 2 we obtain $\frac{\bar{v}_{j+1}^{2}}{4 \bar{\alpha}} \in \mathcal{C}_{2 \cdot 4^{m-j-1}(k+1)^{2(m-j+1)-1}}^{k+1}\left(\mathbf{R}^{n}\right)$ and $\bar{v}_{j+2} \in$ $\mathcal{C}_{4^{m-j-1}(k+1)^{2(m-j)}}^{k+1}\left(\mathbf{R}^{n}\right)$. We also have $\left|\bar{u}_{j+2}\right| \in \mathcal{C}_{4^{m-j-1}(k+1)^{2(m-j)}}^{k+1}\left(\mathbf{R}^{n}\right)$. Indeed, $\left|\bar{u}_{j+2}\right|=\bar{\alpha}-\frac{\bar{v}_{j+1}^{2}}{4 \bar{\alpha}}$

$$
\begin{aligned}
& =\frac{4 \bar{\alpha}^{2}-\bar{v}_{j+1}^{2}}{4 \bar{\alpha}} \\
& =\frac{\bar{\gamma} \bar{A}\left(\bar{\gamma} \bar{A}+2 \bar{v}_{j+1}\right)}{2\left(\bar{\gamma} \bar{A}+\bar{v}_{j+1}\right)}=\frac{\bar{\gamma} \bar{A}}{2} \cdot\left(1+\frac{\bar{v}_{j+1}}{\bar{\gamma} \bar{A}+\bar{v}_{j+1}}\right)=\frac{\bar{\gamma} \bar{A}}{2}+\frac{1}{2} \cdot \frac{\bar{\gamma} \bar{A} \bar{v}_{j+1}}{\bar{\gamma} \bar{A}+\bar{v}_{j+1}} .
\end{aligned}
$$

Now the assertion follows by Lemma 2.

It is clear that $\bar{v}_{j+2}$ and $\left|\bar{u}_{j+2}\right|$ defined above are nonnegative regulous functions on $\mathbf{R}^{n}$ satisfying $\bar{v}_{j+1}^{2}+\left|\bar{u}_{j+2}\right|^{2}=\bar{v}_{j+2}^{2}$. Note that $\left|\bar{u}_{j+2}\right|^{-1}(0)=(\bar{\gamma})^{-1}(0)$ is an algebraic subset of $\mathbf{R}^{n}$. It is also not difficult to observe that if $\left.\bar{\gamma}\right|_{M},\left.\bar{A}\right|_{M}$ and $\left.\bar{v}_{j+1}\right|_{M}$ approximate $\left.\sqrt{v_{j+2}-v_{j+1}}\right|_{M}=\left.f\right|_{M},\left.\left(\sqrt{v_{j+2}-v_{j+1}}+\sqrt{v_{j+2}+v_{j+1}}\right)\right|_{M}$ and $\left.v_{j+1}\right|_{M}$, respectively, with precision high enough, then $\bar{v}_{j+2},\left|\bar{u}_{j+2}\right|$ are good approximations of $v_{j+2},\left|u_{j+2}\right|$ on $M$.

To complete the step number $j+1$ it remains to recover $\bar{u}_{j+2}$ approximating $u_{j+2}$ on $M$ when $\left|\bar{u}_{j+2}\right|$ approximating $\left|u_{j+2}\right|$ is given. To do this, it is sufficient to define 
the sign of $\bar{u}_{j+2}$ on every connected component of $\mathbf{R}^{n} \backslash\left|\bar{u}_{j+2}\right|^{-1}(0)$ and to check that the obtained function satisfies the requirements.

From the equation $\left|\bar{u}_{j+2}\right|=\frac{\bar{\gamma} \bar{A}}{2} \cdot\left(1+\frac{\bar{v}_{j+1}}{\bar{\gamma} \bar{A}+\bar{v}_{j+1}}\right)$ above we know that the zeroes of $\left|\bar{u}_{j+2}\right|$ are precisely the zeroes of $\bar{\gamma}$ (where $\bar{\gamma}$ is the polynomial approximating $f$ on $\bar{G}$ obtained by Lemma 4 ). Let $A_{1}, \ldots, A_{s}$ denote all connected components of $G \backslash f^{-1}(0)$. By Lemma 4 (recall that $g:=1$ ), there is a tubular neighborhood $T \subset \subset G$ of $f^{-1}(0)$ and there are connected components $B_{1}, \ldots, B_{s}$ of $G \backslash(\bar{\gamma})^{-1}(0)$ with $A_{i} \subset B_{i} \cup \bar{T}$ and such that $B_{i} \neq B_{j}$ if $i \neq j$. (Here recall that we assume $\varepsilon<1$ so the polynomial approximating $g$, obtained by applying Lemma 4, does not vanish at any point of $\bar{G}$.)

Recall that $u_{j+2}^{-1}(0) \cup \Sigma=f^{-1}(0)$ and set $\operatorname{sgn}\left(\left.\bar{u}_{j+2}\right|_{B_{i}}\right)=\operatorname{sgn}\left(\left.u_{j+2}\right|_{A_{i}}\right)$, for $i=1, \ldots, s$. On every component of $G \backslash(\bar{\gamma})^{-1}(0)$ different from $B_{1}, \ldots, B_{s}$ set the sign of $\bar{u}_{j+2}$ arbitrarily and set $\left.\bar{u}_{j+2}\right|_{(\bar{\gamma})^{-1}(0)}=0$. Since $(\bar{\gamma})^{-1}(0) \cap \partial G=\emptyset$, there is precisely one connected component $C$ of $G \backslash(\bar{\gamma})^{-1}(0)$ with $\bar{C} \cap \partial G \neq \emptyset$. Therefore on every connected component $E$ of $\left(\mathbf{R}^{n} \backslash G\right) \backslash(\bar{\gamma})^{-1}(0)$ we can define $\operatorname{sgn}\left(\left.\bar{u}_{j+2}\right|_{E}\right)=$ $\operatorname{sgn}\left(\left.\bar{u}_{j+2}\right|_{C}\right)$. Clearly, $\bar{u}_{j+2}$ is a quasi-regulous function and, by Lemma $3, \bar{u}_{j+2} \in$ $\mathcal{C}^{k+1}\left(\mathbf{R}^{n}\right)$.

Let us check that $\bar{u}_{j+2}$ approximates $u_{j+2}$ on $B_{i} \cap M$, for $i=1, \ldots, s$. It is clear that $\bar{u}_{j+2}$ approximates $u_{j+2}$ on $A_{i} \cap B_{i} \cap M$. This is because $u_{j+2}$ and $\bar{u}_{j+2}$ have the same sign at every point of $A_{i} \cap B_{i}$ and $\left|\bar{u}_{j+2}\right|$ approximates $\left|u_{j+2}\right|$ on $M$. On the other hand, $B_{i} \backslash A_{i} \subset \bar{T}$. If this was not true, then we had $\left(\left(B_{i} \backslash A_{i}\right) \backslash \bar{T}\right) \cap A_{j} \neq \varnothing$ for some $j \neq i$ because $\bigcup_{i=1}^{s} A_{i} \cup \bar{T}=G$. Then $\left(\left(B_{i} \backslash A_{i}\right) \backslash \bar{T}\right) \cap\left(B_{j} \cup \bar{T}\right) \neq \emptyset$ so $B_{i} \cap B_{j} \neq \emptyset$ for some $j \neq i$, a contradiction. Since $B_{i} \backslash A_{i} \subset \bar{T}$, the function $\bar{u}_{j+2}$ approximates $u_{j+2}$ on $\left(B_{i} \backslash A_{i}\right) \cap M$ because $\left|u_{j+2}\right|_{M \cap \bar{T}}\left|=\left(f \cdot \sqrt{v_{j+2}+v_{j+1}}\right)\right|_{M \cap \bar{T}}$, $\left|\bar{u}_{j+2}\right|_{M \cap \bar{T}} \mid$ approximates $\left|u_{j+2}\right|_{M \cap \bar{T}} \mid$ and, by Lemma $4,\left.\left(f \cdot \sqrt{v_{j+2}+v_{j+1}}\right)\right|_{M \cap \bar{T}}$ may be assumed as close to zero as we wish. Hence, $\left.u_{j+2}\right|_{M \cap \bar{T}}$ and $\left.\bar{u}_{j+2}\right|_{M \cap \bar{T}}$ are close to zero and therefore close to each other (even if $u_{j+2}$ and $\bar{u}_{j+2}$ have different signs at some points of $M \cap \bar{T}$ ).

Let us check that $\bar{u}_{j+2}$ approximates $u_{j+2}$ on $M \backslash \bigcup_{i=1}^{s} B_{i}$. Observe that $G \backslash \bigcup_{i=1}^{s} B_{i} \subset \bar{T}$. This is because $\bigcup_{i=1}^{s} A_{i} \cup \bar{T}=G$ and, for every $i$, we have $A_{i} \subset B_{i} \cup \bar{T}$. Consequently, $M \backslash \bigcup_{i=1}^{s} B_{i} \subset \bar{T}$ and the assertion follows as above. Thus we have proved that $\bar{u}_{j+2}$ approximates $u_{j+2}$ on $M$.

Finally, recall that, by construction, $\bar{u}_{j}^{-1}(0)$ is an algebraic subset of $\mathbf{R}^{n}$, for every $j=1, \ldots, m+1$. Therefore, $\bar{v}_{m+1}^{-1}(0)=\bar{u}_{1}^{-1}(0) \cap \cdots \cap \bar{u}_{m+1}^{-1}(0)$ is an algebraic subset of $\mathbf{R}^{n}$. If the approximation is close enough, then $\bar{v}_{m+1}^{-1}(0) \cap M=\emptyset$ because $v_{m+1}=1$. In particular, every $w_{j}=\left.\frac{\bar{u}_{j}}{\bar{v}_{m+1}}\right|_{M}$ is a $\mathcal{C}^{k}$ quasi-regulous function approximating $\left.u_{j}\right|_{M}$ and $w_{1}^{2}+\cdots+w_{m+1}^{2}=1$.

Acknowledgements I express my gratitude to Professor Wojciech Kucharz, Professor Krzysztof Nowak and Professor Wiesław Pawłucki for comments, remarks and very helpful discussions.

Open Access This article is distributed under the terms of the Creative Commons Attribution 4.0 International License (http://creativecommons.org/licenses/by/4.0/), which permits unrestricted use, distribution, and reproduction in any medium, provided you give appropriate credit to the original author(s) and the source, provide a link to the Creative Commons license, and indicate if changes were made. 


\section{References}

1. Bochnak, J., Coste, M., Roy, M.-F.: Real Algebraic Geometry. Ergeb. Math. Grenzgeb. vol. 36. Springer, Berlin (1998)

2. Bochnak, J., Kucharz, W.: Algebraic approximation of mappings into spheres. Mich. Math. J. 34, 119-125 (1987)

3. Bochnak, J., Kucharz, W.: Realization of homotopy classes by algebraic mappings. J. Reine Angew. Math. 377, 159-169 (1987)

4. Bochnak, J., Kucharz, W.: On real algebraic morphisms into even-dimensional spheres. Ann. Math. 128(2), 415-433 (1988)

5. Fichou, G., Huisman, J., Mangolte, F., Monnier, J.-P.: Fonctions régulues. J. Reine Angew. Math. 718, 103-151 (2016)

6. Fichou, G., Monnier, J.-P., Quarez, R.: Continuous functions on the plane regular after one blowing-up. Math. Z. 285, 287-323 (2017)

7. Kollár, J., Kucharz, W., Kurdyka, K.: Curve-rational functions. Math. Ann. 370, 39-69 (2018)

8. Kollár, J., Nowak, K.: Continuous rational functions on real and p-adic varieties. Math. Z. 279, 85-97 (2015)

9. Kucharz, W.: Rational maps in real algebraic geometry. Adv. Geom. 9, 517-539 (2009)

10. Kucharz, W.: Approximation by continuous rational maps into spheres. J. Eur. Math. Soc. 16, 15551569 (2014)

11. Kucharz, W.: Piecewise-regular maps. Math. Ann. 372, 1545-1574 (2018)

12. Kucharz, W., Kurdyka, K.: From continuous rational to regulous functions. In: Proc. Int. Cong. of Math.-2018 Rio de Janeiro, vol. 2, pp. 737-766

13. Monnier, J.-P.: Semi-algebraic geometry with rational continuous functions. Math. Ann. 372, 10411080 (2018)

14. Zieliński, M.: Approximation of maps into spheres by regulous maps. Arch. Math. 110, 29-34 (2018)

Publisher's Note Springer Nature remains neutral with regard to jurisdictional claims in published maps and institutional affiliations. 